\title{
Effect of Elevated Ketone Body on Maternal and Infant Outcome of Pregnant Women with Abnormal Glucose Metabolism During Pregnancy
}

This article was published in the following Dove Press journal:

Diabetes, Metabolic Syndrome and Obesity: Targets and Therapy

\author{
Meichen Qian' \\ $\mathrm{Na} \mathrm{Wu}{ }^{1,2}$ \\ Ling $\mathrm{Li}^{1}$ \\ Wenshu Yu' \\ Hong Ouyang' \\ Xinyan Liu' \\ Yujing $\mathrm{He} \mathbb{D}^{\prime}$ \\ Abdulrahman Al-Mureish ${ }^{2}$ \\ 'Department of Endocrinology, Shengjing \\ Hospital of China Medical University, \\ Shenyang II 0004, People's Republic of \\ China; ${ }^{2}$ Clinical Skills Practice Teaching \\ Center, Shengjing Hospital of China \\ Medical University, Shenyang II0004, \\ People's Republic of China
}

\begin{abstract}
Ketone bodies are one of the products of fat metabolism which can be used as an alternative energy source for the human body in states of glucose deficiency. Normal pregnant women may develop ketosis due to physiological changes during pregnancy, while pregnant women with abnormal glucose metabolism are more likely to develop ketosis due to abnormal insulin secretion. Animal experiments and clinical studies have shown that exposure to highketone environments during pregnancy is closely related to adverse maternal and infant outcomes. However, there is no unified conclusion on whether ketone bodies should be routinely monitored during pregnancy. This review summarizes the existing studies on ketone body levels and pregnancy outcomes in the case of abnormal blood glucose during pregnancy, elaborates the current guidelines on the level of ketone bodies, provides the detection and treatment of ketosis in pregnant women with abnormal blood glucose in the clinical practice. Keywords: diabetic ketoacidosis, gestational diabetes mellitus, ketoacidosis, pregestational diabetes mellitus, diabetes mellitus
\end{abstract}

\section{Introduction}

Ketone bodies, including acetoacetate, 3- $\beta$-hydroxybutyrate and acetone, are normal metabolic products of fatty acid breakdown in the liver. The normal ketone bodies levels are $<0.5 \mathrm{mM}$ and levels $>1.0 \mathrm{mM}$ is defined as hyperketonemia, and level $>3.0$ $\mathrm{mM}$ indicate ketoacidosis. ${ }^{1}$ Certain physiological conditions, such as prolonged exercise, improper diet during pregnancy, or ketogenic diet, result in a mild or moderate increase in ketone body levels in the body. The increase results from insufficient availability of glucose leading to increased breakdown of fat into fatty acids which can be oxidized and decomposed in the liver to form ketone bodies.

In some pathological conditions, when blood glucose is too high, the barrier of glucose utilization in peripheral tissues is aggravated, the ketone bodies obtained by the metabolism of fat in the liver exceed the utilization capacity of peripheral tissues, resulting in high blood ketone body content, which leads to ketosis and ketonuria. ${ }^{2}$ Without timely detection and control, it is easy to develop into diabetic ketoacidosis (DKA), which results in a series of pathophysiological phenomena including acidosis, electrolyte balance disorders, circulatory failure, renal failure, and central nervous system dysfunction.

Abnormal glucose metabolism during pregnancy refers to the state of hyperglycemia that occurs during pregnancy, including gestational diabetes mellitus
Department of Endocrinology, Clinical

Skills Practice Teaching Center, Shenging

Hospital of China Medical University,

Shenyang II0004, People's Republic of

China

Email344I535223@qq.com
Diabetes, Metabolic Syndrome and Obesity: Targets and Therapy 2020:13 458I-4588

4581

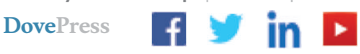

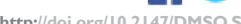


(abnormal glucose metabolism that does not reach the level of overt diabetes during pregnancy, GDM), and overt diabetes in pregnancy (the blood glucose at any time during pregnancy meets the diabetes standard for non-pregnant people), and pregestational diabetes mellitus (diabetes diagnosed before pregnancy, PGDM, including pregestational type-1 diabetes and pregestational type- 2 diabetes). ${ }^{3}$ Studies have shown that pregnant people are more likely to develop ketosis than non-pregnant people. ${ }^{4}$ Affected by multiple risk factors of pregnancy and hyperglycemia, people with abnormal glucose metabolism during pregnancy are at a greater risk of ketosis. This article reviews the impact of ketosis in all kind of abnormal glucose metabolism on pregnancy outcomes, views from different guidelines on ketosis in gestational diabetes, clinical testing methods for ketone bodies, and different opinions about testing ketone in pregnancy.

\section{Ketosis in Abnormal Glucose Metabolism During Pregnancy}

To meet the needs of fetal growth and development, normal pregnant women undergo a series of changes in glucose metabolism, including increased insulin resistance in surrounding tissues, increased glucose excretion and utilization, increased liver gluconeogenesis in the third trimester of pregnancy, and accelerated lipolysis. ${ }^{1}$ Among them, increased glucose utilization and excretion lead to a faster rate of reduction of pregnant women's fasting blood glucose, which is the physiological basis for normal pregnant women to easily develop starvation ketosis when fasting for a long time. Paterson et al studied the blood ketone and urine ketone values of 22 healthy pregnant women and reported that the average blood ketone of pregnant women was much higher than that of non-pregnant women, which proves that pregnant women are more likely to develop ketosis than non-pregnant women. ${ }^{4}$

In 1993, M W Carpenter suggested in a review of metabolic changes in gestational diabetes that pregnant women with GDM were more likely to develop ketosis at the same weight. ${ }^{5}$ Sara L White's team compared metabolic profiles of 646 obese pregnant women, including 198 pregnant women who developed GDM during pregnancy, and found that pregnant women with GDM had higher ketone metabolism than those without GDM. ${ }^{6}$ This maybe relevant to the function of pancreatic $\beta$ cells is impaired and cannot secrete sufficient insulin, gestational impaired glucose tolerance including GDM occurs. ${ }^{7}$
Insulin deficiency can lead to increase lipolysis and disturbance of fat metabolism, which leads to increase plasma free ketone bodies, and easily results in hyperketemia and ketonuria. ${ }^{8}$ For pregnant patients with abnormal glucose metabolism, nutritional therapy is the main clinical treatment. Improper dietary intake results in excessive restrictions on energy intake and increases the probability of starvation ketosis. The risk of starvation ketosis is increased by vomiting caused by early pregnancy reactions and uterine compression in the third trimester leading to insufficient energy intake. ${ }^{2}$ These mechanisms indicate that women with abnormal glucose metabolism during pregnancy have a greater risk of ketosis than normal pregnant women as confirmed earlier. Under the premise of equal weight, pregnant women with GDM are more likely to develop ketosis than normal pregnant women. Studies have also shown that the ketone body content of pregnant women with PGDM was higher than that of normal pregnant women, and pregnant women with normal OGTT had lower levels of ketosis than pregnant women with confirmed GDM between 25 and 37 weeks of pregnancy. ${ }^{9}$ These studies show that abnormal glucose metabolism during pregnancy can affect the incidence of ketosis.

\section{Mechanism of Adverse Outcomes Caused by Ketonemia}

Multiple studies including animal experiments have shown that high-ketone environment may cause adverse outcomes for both mother and fetal, although the mechanism is still unclear. ${ }^{10,11}$ Mouse embryos exposed to $\beta$-hydroxybutyric acid artificially exhibited abnormalities including developmental slowing and abnormal neural tube closure, and earlier the embryo, the higher the exposure dose and the incidence of deformity. ${ }^{11}$ Animal models exposed to highketone levels during pregnancy exhibited malformations of larger hearts, larger total embryonic volumes, and impaired brain development compared with controls with normal ketone bodies. ${ }^{10}$ But early studies have pointed out that the teratogenic level of blood ketone bodies in animal models is 20 to 40 times that of normal conditions, it may be difficult to achieve in the case of ketoacidosis. ${ }^{12}$ However, a review in 2015 on ketoacidosis during diabetic pregnancy suggested that the possible harm of ketone bodies to the fetus should be discussed in conjunction with the effects of ketone bodies on different organs of the fetus in non-extreme situations and the pathomechanisms 
underlying these effects. ${ }^{13}$ Existing physiological studies have shown that ketone body levels are associated with embryonic brain structural development ${ }^{14}$ glucose uptake by cardiomyocytes, ${ }^{15}$ tubular cell growth, ${ }^{16}$ and validated markers in blood vessels. ${ }^{17}$ There are currently no animal trials simulating the adverse effects of hyperketonemia on the mother and offspring in models of gestational dysglycemia. In the case of both abnormal blood glucose and ketone bodies during pregnancy, which aspects of maternal and offspring adverse outcomes are more affected deserve to be discussed and studied.

\section{Relationship Between Abnormal Ketone Body Levels and Different Adverse Pregnancy Outcomes}

Uncontrolled blood glucose abnormalities during pregnancy leads to a variety of adverse pregnancy outcomes. Studies have shown that poor blood glucose control in GDM is associated with macrosomia, neonatal hypoglycemia, neonatal jaundice, and congenital malformations of multiple organs. ${ }^{18}$ At the same time, the state of hyperglycemia during pregnancy is also considered to be closely related to a variety of complications during childbirth, and the rate of caesarean section. However, there are not many studies on the relationship between ketosis caused by abnormal blood glucose in pregnancy and adverse pregnancy outcomes.

\section{Ketone Bodies and Congenital Malformations}

As early as 1989, an animal experiment had proposed that $\beta$ hydroxybutyric acid had a synergistic effect with glucose, affecting embryo growth and inducing embryonic malformations. ${ }^{19}$ The researches on the correlation between the ketone body content and fetal malformations conducted by Qingxin et al and Xinyuan et al in 2016 and 2018 respectively showed that the higher the ketone body content, higher the risk of congenital malformations in the offspring. ${ }^{20,21}$ Fetal malformations that are statistically correlated with ketone body content in the two studies include cleft lip and cleft palate, cardiovascular system malformations, nervous system malformations (such as anencephaly and spina bifida), and digestive system malformations.

\section{Ketone Bodies and Impaired Central Nervous System Development}

Under normal physiological conditions, ketone bodies play an indispensable physiological role in the central nervous system, including the brain, but there are evidences that exposure to high-ketone environments during pregnancy can affect the neural development of the fetus, and this effect still exists after delivery. Rizzo et al tracked the post-birth conditions of 196 singleton offspring of pregnant women (95 of them with pregestational diabetes mellitus, 101 with gestational diabetes mellitus), and used the Bayley Scales of Infant and Toddler Development and Bruininks-Oseretsky Test of Motor Proficiency for the offspring at the age of 2 and 6 to 9 years. Results showed that the average scores of the offspring in two tests were inversely proportional to the mother's $\beta$-hydroxybutyric acid content during pregnancy and childbirth. ${ }^{22}$ In another study on the neuropsychological development of the offspring of diabetic mothers, Rizzo et al collected the offspring's scores of the Neonatal Behavioral Assessment Scale and the StanfordBinet Intelligence Scale, compared them with biochemical indicators of diabetic mothers during pregnancy, and found that children's IQ was negatively correlated with the $\beta$ hydroxybutyrate content of pregnant women in the second trimester. $^{23}$

\section{Ketone Bodies and Macrosomia}

Macrosomia is defined as a birth weight greater than or equal to $4000 \mathrm{~g}$, and both hyperglycemia and hyperinsulinemia lead to increase fat storage in the body of the fetus, which results in an increase in fetal weight to produce macrosomia. ${ }^{24}$ A retrospective study including 1981 cases of pregnant women with GDM and associated macrosomia analyzed the risk factors of GDM, ketone bodies and highdensity lipoprotein, triglycerides were found to be significant indicators of GDM with macrosomia. ${ }^{25}$ The specific physiological mechanism by which ketone bodies cause macrosomia is not known for the time being, perhaps related to the fact that hyperglycemia predisposes to ketosis.

\section{Ketone Bodies and Complications During Childbirth}

In 2018, Huang et al monitored and recorded the urinary ketones and delivery results of 570 pregnant women with GDM during childbirth and found that maternal ketonuria is quite common in women with GDM who have not given birth. ${ }^{26}$ According to the different urine ketone content, 570 subjects were divided into three groups: negative, moderate ketonuria and ketosis. By comparing the birth complications between different urine ketone levels, 
Huang SY et al found that incidences of FHR III, thirddegree amniotic fluid contamination, and postpartum hemorrhage increased with the development of ketonuria. At the same time, the pregnant women in the ketosis group with the most severe ketonuria took significantly longer time to give birth than the women in the other two groups, and had a higher rate of operative vaginal delivery. Researchers believe that the possible reason is the metabolic fuel in pregnant women with ketonuria is not glucose but lipids, and studies have shown that the lack of glucose during childbirth increases the delivery time compared to pregnant women with sufficient glucose. ${ }^{27}$ The increase in the rate of operative vaginal delivery is believed to be related to the increase in other adverse complications during delivery.

\section{Monitoring and Treatment of Ketone Bodies in Patients with Abnormal Glucose Metabolism During Pregnancy}

\section{Methods for Detecting Ketone Bodies}

The methods for detecting ketone bodies include the urine test, and the blood test for ketones. The urine test for ketones is based on the principle of sodium nitroprusside reaction which is commonly used in the outpatient department. Since sodium nitroprusside only reacts with acetoacetate and acetone, the content of $\beta$-hydroxybutyric acid (which is the predominant metabolism in ketosis) is not expressed. But the ratio of acetoacetic acid to $\beta$-hydroxybutyric acid in the human body is about $1: 10$, cause the quantity of ketones measured in urine does not represent the true plasm ketone concentration. Meanwhile, urine ketone measurement is affected by urine output and the average of concentration within the urine held in the bladder. Finally, urine reading can still rise even the acidosis disappears due to the oxidation between blood $\beta$-hydroxybutyric and acetoacetate. $^{28}$ These make the urine test for ketones, which uses acetoacetate and acetone in urine as the detection materials, has a certain hysteresis. At the same time, the concentration of ketone bodies in urine reflects the content of all ketone bodies accumulated since the last urination of the human body, thereby the urine test for ketones is affected by the patient's urine output. ${ }^{29}$

Blood ketones are not affected by drugs or renal function hence samples can be reserved without lag, which is conducive to rapid disease diagnosis, and thus the blood test for ketones has gradually been recognized in clinical practice. ${ }^{30}$ The blood test for ketones includes the enzymatic method of measuring $\beta$-hydroxybutyric acid in plasma or serum with a fully automatic biochemical analyzer and the electrochemical method of measuring $\beta$ hydroxybutyric acid in capillary blood with a blood ketone meter. ${ }^{2}$ Studies have shown that the level of $\beta$-hydroxybutyric acid measured in the capillaries is significantly correlated with the level of serum ketones, and the peripheral blood ketone levels measured in the capillaries can be regarded as the real-time levels of blood ketones in the body. ${ }^{31}$ The "Expert Consensus on Blood Ketone Monitoring for Diabetes in China" recommends that women with GDM should be monitored for blood ketones during dietary treatment, daily blood glucose monitoring, and early pregnancy when the early pregnancy reaction is strong. ${ }^{2}$

Ketone should be monitored after meals. Fasting monitoring may lead to false-positive. ACOG guidelines suggest that pregnant women with prenatal diabetes should monitor ketones when their blood glucose reaches $200 \mathrm{mg}$ / $\mathrm{dL}$ to prevent DKA. ${ }^{32}$ Other guidelines mostly recommend that ketone bodies be monitored immediately when there is a suspected risk of DKA.

\section{Recommendations of Various Guidelines for Monitoring Ketone Bodies During Pregnancy}

At present, the guidelines of various diabetes associations worldwide all mention ketosis in the management of gestational diabetes, but the focuses are different (The specific content is shown in Table 1). ${ }^{32-35}$ Among them, the guidelines of the American Diabetes Association, the American College of Obstetricians and Gynecologists, and the Joint British Diabetes Societies focus more on the prevention of diabetic acidosis, while the International Federation of Obstetrics and Gynecology and the Chinese Diabetes Society focus more on the relationship between the diet control and the occurrence of starvation ketosis to discuss the monitoring of ketone bodies.

\section{Treatment of Ketone Bodies in Abnormal Glucose Metabolism}

The methods of blood glucose control during pregnancy mainly include diet control, moderate exercise, and medication to reduce blood glucose levels. The "Chinese guidelines for the prevention and treatment of T2DM state" proposed that the blood glucose control of patients 
Table I The Different Focuses of Guidelines for Ketosis in Gestational Diabetes

\begin{tabular}{|c|c|c|c|}
\hline Focuses & Guidelines & Standards for Testing Ketone Bodies & $\begin{array}{l}\text { Aiming Type of } \\
\text { Diabetes }\end{array}$ \\
\hline \multirow[t]{3}{*}{$\begin{array}{l}\text { Ketone content and } \\
\text { prevention of DKA }\end{array}$} & $\begin{array}{l}\text { ADA } \\
\text { guidelines } \\
(2020)^{33}\end{array}$ & $\begin{array}{l}\text { Pregnancy is a ketogenic state. Women with type I diabetes (and a small } \\
\text { number of women with type } 2 \text { diabetes) with lower blood glucose are more } \\
\text { likely to develop diabetic ketoacidosis (DKA) than when they are not pregnant. } \\
\text { Women with type I diabetes should use urine ketone test strips to monitor } \\
\text { their ketone body status and receive education on the prevention and } \\
\text { monitoring of diabetic ketoacidosis. }\end{array}$ & Type I diabetes \\
\hline & $\begin{array}{l}\text { ACOG } \\
\text { guidelines } \\
(2018)^{32}\end{array}$ & $\begin{array}{l}\text { In order to prevent the occurrence of DKA, women with diabetes before } \\
\text { pregnancy should monitor urine ketones when their blood glucose reaches } 200 \\
\mathrm{mg} / \mathrm{dL} \text {. } \\
\text { Women with type } 2 \text { diabetes are also at a risk of DKA, and the same } \\
\text { monitoring method should be adopted to prevent DKA. }\end{array}$ & Type 2 diabetes \\
\hline & $\begin{array}{l}\text { JBDS } \\
\text { guidelines } \\
(2017)^{34}\end{array}$ & $\begin{array}{l}\text { Pregnant women with type I diabetes who use insulin pumps to control blood } \\
\text { glucose should stop using insulin pumps and start variable rate intravenous } \\
\text { insulin infusion if urine ketone++ or blood ketone value is higher than } 1.5 \mathrm{mmol} / \\
\mathrm{L} \text { during delivery. } \\
\text { Pregnant women on metformin or multiple daily injections should check Urea } \\
\text { and electrolytes every } 4-6 \mathrm{~h} \text { during labour to maintain safe levels of potassium } \\
\text { and bicarbonate. Blood ketones should be checked if ketoaci- dosis is suspected. } \\
\text { The early stage of ketoacidosis may only be accompanied by a slight increase in } \\
\text { blood glucose or nausea and abdominal pain. Therefore, it is recommended that } \\
\text { DKA should be taken into consideration for pregnant women with diabetes } \\
\text { when they are unwell, and urine ketones or blood ketones should be measured } \\
\text { to exclude DKA. }\end{array}$ & $\begin{array}{l}\text { Type I diabetes and } \\
\text { pregnancy with insulin } \\
\text { injection }\end{array}$ \\
\hline \multirow[t]{2}{*}{$\begin{array}{l}\text { Ketone bodies and } \\
\text { starvation ketosis }\end{array}$} & $\begin{array}{l}\text { FIGO } \\
\text { guidelines } \\
(2015)^{35}\end{array}$ & $\begin{array}{l}\text { The incidence of ketosis in pregnant women increases when the calorie intake } \\
\text { is lower than I } 500 \mathrm{kcal} \text { per day, and the control of } 1600 \text { to } 1800 \mathrm{kcal} \text { per day will } \\
\text { not lead to an increase in ketone bodies. } \\
\text { No mention of ketone detection. }\end{array}$ & All pregnant women \\
\hline & $\begin{array}{l}\text { CDS } \\
\text { guidelines } \\
(2017)^{2}\end{array}$ & $\begin{array}{l}\text { The standard of dietary control during pregnancy should not cause starvation } \\
\text { ketosis while ensuring energy supply of mother and child and maintaining a } \\
\text { normal blood glucose level. } \\
\text { Encourage pregnant women to perform blood glucose self-monitoring, } \\
\text { including checking fasting and postprandial blood glucose levels, and checking PG } \\
\text { and urine ketone bodies I 2 hours after meal. } \\
\text { Blood glucose level should be checked when urine ketone bodies are positive, if } \\
\text { normal, starvation ketosis should be considered, and food intake should be } \\
\text { increased in time. }\end{array}$ & All pregnant women \\
\hline
\end{tabular}

Abbreviations: ADA, American Diabetes Association; ACOG, American College of Obstetricians and Gynecologists; FIGO, The International Federation of Gynecology and Obstetrics; JBDS, Joint British Diabetes Societies; CDS, Chinese Diabetes Society.

with GDM should avoid oral hypoglycemic drugs as much as possible, and insulin therapy should be performed when the dietary treatment on blood glucose control is not effective. Pregnant women with abnormal blood glucose have more complex dietary requirements than normal pregnant women because they need to ingest enough macroelements and trace elements for fetal development while limiting postprandial blood glucose growth. Among the nutrients, carbohydrate intake has the greatest impact on postprandial blood glucose, and glucose from carbohydrates is also the main energy substrate for placenta and fetus. The latest literature suggests that the carbohydrate content intake of pregnant women with abnormal blood glucose should be individualized by clinical dietitians, and attention should be paid to the type of carbohydrates, and starch foods with higher dietary fiber should be selected as the main food. ${ }^{36}$ 
If dietary education is ineffective and women with abnormal blood glucose in pregnancy fail to follow or adjust their dietary plan without authorization, they may have abnormal blood glucose or blood ketones caused by improper diet. However, some scholars consider that diet control during pregnancy, especially the restriction of carbohydrate intake, will increase the possibility of ketosis. According to the 2017 "International Diabetes Federation Diabetes Atlas", approximately $16.2 \%$ of pregnant women worldwide have carbohydrate disorders during pregnancy, and $75 \%$ to $90 \%$ of them are GDM patients. ${ }^{37} \mathrm{~A}$ randomized controlled trial involving 436 obese pregnant women found that levels of $\beta$-hydroxybutyric acid and fatty acids in pregnant women who had undergone dietary intervention increased, that is, decreased carbohydrate intake would lead to an increase in fat metabolism that can produce ketone bodies. ${ }^{38}$ A study in 2015 compared the blood ketone values and metabolism states of 180 women with gestational diabetes, and found that the weight loss of pregnant women was correlated with $\beta$ hydroxybutyric acid. ${ }^{27}$ However, from 2016 to 2018, Mijatovic et al randomly divided 46 women with gestational diabetes to the low-carbohydrate group and the routine care group, and found there was no significant differences in the blood ketone values and pregnancy outcomes between the two groups of pregnant women. ${ }^{39}$ Therefore, whether dietary control for pregnant women with GDM will lead to the occurrence of ketosis remains to be further studied.

When the increase in ketone bodies are caused by high blood glucose, drugs should be used to reduce the blood glucose level rationally. In China, the first drug of choice for abnormal blood glucose during pregnancy is insulin. Metformin is available but not recommended. Observation of the utilization and removal of ketone bodies through tracer technology found that insulin can effectively reduce the content of ketone bodies in three aspects: inhibiting lipolysis, inhibiting the production of ketone bodies in the liver, and accelerating the use of ketones by surrounding tissues. ${ }^{40}$ There is no definite evidence of the effect of metformin on the content of ketone bodies in the body under the condition of abnormal blood glucose.

When pregnant women with abnormal blood glucose are found to have elevated ketone bodies, the ketone symptoms of pregnant women should be relieved first to avoid the development of ketoacidosis. After controlling the blood ketone body to the normal range, the causes of ketone body elevation should be actively searched for and disposed of separately according to the causes. If it is hunger ketosis related to dietary control, pregnant women should be evaluated for the daily intake of nutrients, adjust the dietary program, and carry out health education related to dietary control for pregnant women. If ketone body elevation is caused by hyperglycemia, adjust the current hypoglycemic regimen and monitor the fasting and postprandial blood glucose of pregnant women until the blood glucose level of pregnant women is controlled within normal range.

\section{Conclusion}

With the improvement of living standards, the incidence of GDM has been on the rise in recent years, and the adverse effects of high glucose in pregnancy on pregnant women and fetuses have also paid more and more attention. A number of clinical studies have proved that high ketone bodies in women with GDM are associated with various adverse pregnancy outcomes. Therefore, clinicians should pay extra attention to the patient's ketone body level when performing regular check-ups on women with gestational diabetes, and provide health education to pregnant women about the hazards of elevated ketone bodies. Mild transient ketosis will not cause great harm to pregnant women under timely treatment, while ketoacidosis can endanger the health and life of pregnant women. However, the effects of moderate to severe ketosis that has not reached ketoacidosis and long-term mild ketosis on pregnant women and fetuses need to be further studied.

\section{Statement of Ethics}

This article does not contain any studies with human or animals performed by any of the authors.

\section{Consent for publication}

Agreed to publish.

\section{Acknowledgments}

We gratefully acknowledge Haitao Shen for providing intellectual support and technical assistance.

\section{Author Contributions}

Meichen Qian devised the project, collect material and wrote the manuscript. $\mathrm{Na} \mathrm{Wu}$, Ling Li helped with the discussion, supervised the project and provided critical feedback. All authors made substantial contributions to conception and design, acquisition of data, or analysis and interpretation of data; took part in drafting the article 
or revising it critically for important intellectual content; gave final approval of the version to be published; and agree to be accountable for all aspects of the work.

\section{Funding}

This research was supported by the National Natural Science Foundation of China (81700706), the Clinical Research Project of Liaoning Diabetes Medical Nutrition Prevention Society (LNSTNBYXYYFZXH-RS01B) and the 345 Talent Project of Shengjing Hospital.

\section{Disclosure}

The authors declare no conflicts of interest in this work.

\section{References}

1. Laffel L. Ketone bodies: a review of physiology, pathophysiology and application of monitoring to diabetes. Diabetes Metab Res Rev. 1999;15 (6):412-426. doi:10.1002/(SICI)1520-7560(199911/12)15:6<412:: AID-DMRR72>3.0.CO;2-8

2. Chinese Society of Endocrinology. Expert consensus on blood ketone monitoring for diabetes in China. Chin J Endocrinol Metab. 2014;30 (3):177-183.

3. Chinese Diabetes Society. Chinese guidelines for the prevention and treatment of T2DM state (2017 edition). Chin J Pract Intern Med. 2018;38(04):292-344.

4. Paterson P, Sheath J, Taft P, Wood C. Maternal and foetal ketone concentrations in plasma and urine. Lancet. 1967;1(7495):862-865. doi:10.1016/S0140-6736(67)91426-2

5. Carpenter MW. Metabolic changes in gestational diabetes. Clin Perinatol. 1993;20(3):583-591. doi:10.1016/S0095-5108(18)30386-5

6. White SL, Pasupathy D, Sattar N, et al. Metabolic profiling of gestational diabetes in obese women during pregnancy. Diabetologia. 2017;60(10):1903-1912. doi:10.1007/s00125-0174380-6

7. Baz B, Riveline JP, Gautier JF. Endocrinology of pregnancy: gestational diabetes mellitus: definition, aetiological and clinical aspects. Eur J Endocrinol. 2016;174(2):R43-51. doi:10.1530/EJE-15-0378

8. Danian Z, Tinghuai W. Physiology. 8th ed. People's Medical Publishing House; 2013.

9. Gin H, Vambergue A, Vasseur C, et al. Blood ketone monitoring: a comparison between gestational diabetes and non-diabetic pregnant women. Diabetes Metab. 2006;32(6):592-597. doi:10.1016/S12623636(07)70313-0

10. Sussman D, van Eede M, Wong MD, Adamson SL, Henkelman M. Effects of a ketogenic diet during pregnancy on embryonic growth in the mouse. BMC Pregnancy Childbirth. 2013;13:109. doi:10.1186/ 1471-2393-13-109

11. Horton WE, Sadler TW. Effects of maternal diabetes on early embryogenesis. Alterations in morphogenesis produced by the ketone body, B-hydroxybutyrate. Diabetes. 1983;32(7):610-616. doi:10.2337/ diab.32.7.610

12. Jovanovic L, Metzger BE, Knopp RH, et al. The diabetes in early pregnancy study: beta-hydroxybutyrate levels in type 1 diabetic pregnancy compared with normal pregnancy. NICHD-diabetes in early pregnancy study group (DIEP). National institute of child health and development. Diabetes Care. 1998;21(11):1978-1984. doi:10.2337/diacare.21.11.1978
13. Dalfrà $\mathrm{M}$, Burlina $\mathrm{S}$, Sartore $\mathrm{G}$, Lapolla $\mathrm{A}$. Ketoacidosis in diabetic pregnancy. J Matern Fetal Neonatal Med. 2016;29(17):2889-2895.

14. Sussman D, Ellegood J, Henkelman M. A gestational ketogenic diet alters maternal metabolic status as well as offspring physiological growth and brain structure in the neonatal mouse. $B M C$ Pregnancy Childbirth. 2013;13:198. doi:10.1186/1471-2393-13-198

15. Pelletier A, Coderre L. Ketone bodies alter dinitrophenol-induced glucose uptake through AMPK inhibition and oxidative stress generation in adult cardiomyocytes. Am J Physiol Endocrinol Metab. 2007;292(5):E1325-1332. doi:10.1152/ajpendo.00186.2006

16. Guh JY, Chuang TD, Chen HC, et al. Beta-hydroxybutyrate-induced growth inhibition and collagen production in HK-2 cells are dependent on TGF-beta and Smad3. Kidney Int. 2003;64(6):2041-2051. doi:10.1046/j.1523-1755.2003.00330.x

17. Rains JL, Jain SK. Hyperketonemia increases monocyte adhesion to endothelial cells and is mediated by LFA-1 expression in monocytes and ICAM-1 expression in endothelial cells. Am J Physiol Endocrinol Metab. 2011;301(2):E298-306. doi:10.1152/ajpendo.00038.2011

18. Guanghua W, Juanli W, Miaomiao Z, Jie Z, Liling Z. Association of gestational diabetes mellitus with adverse neonatal outcomes: a metaanalysis. J Tongji Univ. 2016;1:65-72.

19. Sadler TW, Hunter ES, Wynn RE, Phillips LS. Evidence for multifactorial origin of diabetes-induced embryopathies. Diabetes. 1989;38(1):70-74. doi:10.2337/diab.38.1.70

20. Xinyuan Z, Jianhua Z, Jin Z. Correlation between changes of ketone body and inositol content in gestational diabetes mellitus patients and its correlation with fetal malformation. Chin J Birth Health Heredity. 2018;26(06):64-66.

21. Qingxin Q, Zhenghua X, Xiaobin X. Application of three dimensional visualization technology for laparoscopic Resection of Followup study of gestational diabetes mellitus female ketone and inositol content and fetal malformation. J Mol Imaging. 2016;39(3):229-231.

22. Rizzo TA, Dooley SL, Metzger BE, Cho NH, Ogata ES, Silverman BL. Prenatal and perinatal influences on long-term psychomotor development in offspring of diabetic mothers. Am $J$ Obstet Gynecol. 1995;173(6):1753-1758. doi:10.1016/0002-9378(95) 90422-0

23. Silverman BL, Rizzo T, Green OC, et al. Long-term prospective evaluation of offspring of diabetic mothers. Diabetes. 1991;40 (Suppl 2):121-125. doi:10.2337/diab.40.2.S121

24. Kc K, Shakya S, Zhang H. Gestational diabetes mellitus and macrosomia: a literature review. Ann Nutr Metab. 2015;66(Suppl 2):14-20. doi:10.1159/000371628

25. Kang X, Liang Y, Wang S, et al. Prediction model comparison for gestational diabetes mellitus with macrosomia based on risk factor investigation. J Matern Fetal Neonatal Med. 2019;1-10.

26. Huang SY, Yu B, He X, Chen Y. Intrapartum results on differing degrees of ketonuria in nulliparous women with Gestational diabetes mellitus during spontaneous labor. Int $J$ Endocrinol. 2019;2019:7207012. doi:10.1155/2019/7207012

27. Spanou L, Dalakleidi K, Zarkogianni K, et al. Ketonemia and ketonuria in gestational diabetes mellitus. Hormones (Athens, Greece). 2015;14(4):644-650.

28. Dhatariya K. Blood ketones: measurement, interpretation, limitations, and utility in the management of diabetic ketoacidosis. Rev Diabet Stud. 2016;13(4):217-225. doi:10.1900/RDS.2016.13.217

29. Misra S, Oliver NS. Utility of ketone measurement in the prevention, diagnosis and management of diabetic ketoacidosis. Diabet Med. 2015;32(1):14-23. doi:10.1111/dme.12604

30. Hua J, Min L, Cong W, et al. Comparison of the clinical significance of capillary blood $\beta$-hydroxybutyrate and urine ketone in the diagnosis and treatment of diabetic ketosis. Prog Mod Med. 2016;16 (15):2872-2875. 
31. Lertwattanarak R, Plainkum P. Efficacy of quantitative capillary betahydroxybutyrate measurement in the diagnosis of diabetic ketoacidosis: a comparison to quantitative serum ketone measurement by nitroprusside reaction. J Med Assoc Thai. 2014;97(Suppl 3):S78-85.

32. Gestational Diabetes Mellitus. ACOG practice bulletin No. 190: American college of obstetricians and gynecologists. Obstet Gynecol. 2018;131(2):e49-e64. doi:10.1097/AOG.0000000000002501

33. American Diabetes Association. 14. Management of diabetes in pregnancy: standards of medical care in diabetes-2020. Diabetes Care. 2020;43(Suppl1):S183-s192. doi:10.2337/dc20-S014

34. Dashora U, Murphy HR, Temple RC, et al. Managing hyperglycaemia during antenatal steroid administration, labour and birth in pregnant women with diabetes. Diabet Med. 2018;35(8):1005-1010. doi:10.1111/dme. 13674

35. Hod M, Kapur A, Sacks DA, et al. The international federation of gynecology and obstetrics (FIGO) initiative on gestational diabetes mellitus: a pragmatic guide for diagnosis, management, and care. Int J Gynaecol Obstet. 2015;131(Suppl 3):S173-211.

36. Rasmussen L, Poulsen CW, Kampmann U, Smedegaard SB, Ovesen PG, Fuglsang J. Diet and healthy lifestyle in the management of gestational diabetes mellitus. Nutrients. 2020;12(10):3050. doi:10.3390/nu12103050
37. Cho NH, Shaw JE, Karuranga S, et al. IDF diabetes atlas: global estimates of diabetes prevalence for 2017 and projections for 2045 . Diabetes Res Clin Pract. 2018;138:271-281. doi:10.1016/j. diabres.2018.02.023

38. Harreiter J, Simmons D, Desoye G, et al. Nutritional lifestyle intervention in obese pregnant women, including lower carbohydrate intake, is associated with increased maternal free fatty acids, $3-\beta$-hydroxybutyrate, and fasting glucose concentrations: a secondary factorial analysis of the European multicenter, randomized controlled DALI lifestyle intervention trial. Diabetes Care. 2019;42(8):1380-1389.

39. Mijatovic J, Louie JCY, Buso MEC, et al. Effects of a modestly lower carbohydrate diet in gestational diabetes: a randomized controlled trial. Am J Clin Nutr. 2020;112(2):284-292. doi:10.1093/ajcn/ nqaa137

40. Keller U, Lustenberger M, Müller-Brand J, Gerber PP, Stauffacher W. Human ketone body production and utilization studied using tracer techniques: regulation by free fatty acids, insulin, catecholamines, and thyroid hormones. Diabetes Metab Rev. 1989;5(3):285-298. doi:10.1002/dmr.5610050306

Diabetes, Metabolic Syndrome and Obesity: Targets and Therapy

\section{Dovepress}

\section{Publish your work in this journal}

Diabetes, Metabolic Syndrome and Obesity: Targets and Therapy is an international, peer-reviewed open-access journal committed to the rapid publication of the latest laboratory and clinical findings in the fields of diabetes, metabolic syndrome and obesity research. Original research, review, case reports, hypothesis formation, expert opinion and commentaries are all considered for publication. The manuscript management system is completely online and includes a very quick and fair peer-review system, which is all easy to use. Visit http://www.dovepress.com/testimonials.php to read real quotes from published authors.

Submit your manuscript here: https://www.dovepress.com/diabetes-metabolic-syndrome-and-obesity-targets-and-therapy-journal 